\title{
Correspondence
}

\section{Lipoprotein macroaggregates in bronchoalveolar lavage fluid from patients with difiuse interstitial lung disease}

SIR,-I write to propose some factors which may contribute to the formation of lipoprotein macroaggregates of the type recently described by Dr P L Haslam and others (February 1988;43:140-6).

As Dr Haslam and her colleagues have pointed out, large multilamellar aggregates of lipoprotein may be found in the lungs of patients suffering from interstitial lung disease or idiopathic alveolar lipoproteinosis; in addition, patients with idiopathic alveolar lipoproteinosis may have small lamellar bodies and amorphous debris in their lungs.

I have observed similar aggregates in the distal lung spaces of some human fetuses and neonates. When examined in the transmission electron microscope, the aggregates were seen to have an irregular shape and often occupied corners of the terminal saccules. Amorphous material, cellular debris, and numerous lamellar bodies (diameter range 1-8 $\mu \mathrm{m}$ ) were found within the aggregates. Moreover, the aggregates could be displaced by endobronchial lavage and isolated by centrifugation. The staining reactions suggested that they were composed of lipid and lipoprotein. It is perhaps significant that the lungs containing this material were obtained from anencephalic fetuses that had displayed no intercostal or diaphragmatic activity when studied by ultrasonic scan before birth. In all cases death occurred in the period immediately after delivery as the fetuses were incapable of establishing regular breathing. Electron microscopic investigation also confirmed that the lungs of these fetuses had a well differentiated respiratory epithelium and pulmonary capillary network: indeed, the cytological appearances of the type II epithelial cells indicated that they were capable of secreting pulmonary surfactant.

All of the available evidence suggests that alveolar macroaggregates are composed of relatively insoluble material. A mixture of phospholipid (presumably largely derived from pulmonary surfactant), alveolar exudates, and cell debris could also be expected to be viscous. Consequently, it would be difficult to move this material in a proximal direction towards the terminal bronchioles, where the first part of the cilial "escalator" is located. Moreover, local macrophages may well have difficulty in phagocytosing large masses of insoluble and viscous material of this type. For all of these reasons, I suggest that the macroaggregates in fetal and adult lungs are essentially similar in composition and that poor drainage and cleansing of the terminal air spaces may be important factors in their formation.

COWAN MEBAN

Department of Anatomy

Queen's University of Belfast

Belfast BT9 7BL
Anomalous drainage of inferior vena cava to left atrium in association with total anomalous pulmonary venous drainage

SIR,-I remain doubtful of the diagnosis of anomalous drainage of the inferior vena cava to the left atrium in the case report by Mr R K Lamb and associates (November 1987;42:907-8). When all fossa ovalis tissue is absent the atrial septal defect is confluent with the orifice of the inferior vena cava. The eustachian valve of the inferior vena cava then overhangs the atrial septal defect and it can be easily mistaken for septum primum at operation.' If such a mistake is made, the inferior vena cava will drain into the left atrium. The authors have failed to show that the inferior vena cava drained to the left of the septum primum before closure of the atrial septal defect.

\section{NS CHAN \\ Department of Cardiology Green Lane Hospital Auckland \\ New Zealand}

1 Kirklin JW, Barratt-Boyes BG. Cardiac surgery. Chichester: John Wiley and Sons, 1986.

**This letter was sent to the authors, who reply below.

SIR,-We are grateful for Dr Chan's comments about our paper. We accept his criticism but wish to clarify that at reoperation the inferior margin of the tissue excised, referred to as "atrial septum," was thick and muscularised. This was therefore true atrial septum and not eustachian valve.

RK LAMB Wessex Cardiothoracic Centre Southampton General Hospital Southampton SO9 $4 X Y$

SA QURESHI DI HAMILTON Royal Liverpool Children's Hospital Liverpool L7 7DG

RG PATEL Royal Manchester Children's Hospital Manchester M27 IHA 